\title{
The survival time of chocolates on hospital wards: covert observational study
}

\author{
(c) $(1) \Theta$ OPEN ACCESS
}

\section{Parag R Gajendragadkar cardiology specialist registrar ${ }^{1}$, Daniel J Moualed ENT surgery specialist registrar $^{2}$, Phillip L R Nicolson haematology specialist registrar ${ }^{3}$, Felicia D Adjei core medical trainee ${ }^{1}$, Holly E Cakebread foundation year doctor ${ }^{1}$, Rudolf M Duehmke cardiology specialist registrar ${ }^{1}$, Claire A Martin cardiology specialist registrar ${ }^{1}$}

${ }^{1}$ Department of Cardiology, Bedford Hospital, Bedford MK42 9DJ, UK; ${ }^{2}$ Department of Ear, Nose and Throat Surgery, Great Western Hospital, Swindon, UK; ${ }^{3}$ Department of Haematology, University Hospital of North Staffordshire, Stoke-on-Trent, UK

\begin{abstract}
Objective To quantify the consumption of chocolates in a hospital ward environment.

Design Multicentre, prospective, covert observational study.

Setting Four wards at three hospitals (where the authors worked) within the United Kingdom.

Participants Boxes of Quality Street (Nestlé) and Roses (Cadbury) on the ward and anyone eating these chocolates.

Intervention Observers covertly placed two $350 \mathrm{~g}$ boxes of Quality Street and Roses chocolates on each ward (eight boxes were used in the study containing a total of 258 individual chocolates). These boxes were kept under continuous covert surveillance, with the time recorded when each chocolate was eaten.

Main outcome measure Median survival time of a chocolate.

Results 191 out of 258 (74\%) chocolates were observed being eaten. The mean total observation period was 254 minutes (95\% confidence interval 179 to 329). The median survival time of a chocolate was 51 minutes (39 to 63). The model of chocolate consumption was non-linear, with an initial rapid rate of consumption that slowed with time. An exponential decay model best fitted these findings (model $R^{2}=0.844$, $P<0.001$ ), with a survival half life (time taken for $50 \%$ of the chocolates to be eaten) of 99 minutes. The mean time taken to open a box of chocolates from first appearance on the ward was 12 minutes (95\% confidence interval 0 to 24). Quality Street chocolates survived longer than Roses chocolates (hazard ratio for survival of Roses $v$ Quality Street $0.70,95 \%$ confidence interval 0.53 to $0.93, P=0.014$ ). The highest
\end{abstract}

percentages of chocolates were consumed by healthcare assistants $(28 \%)$ and nurses $(28 \%)$, followed by doctors $(15 \%)$.

Conclusions From our observational study, chocolate survival in a hospital ward was relatively short, and was modelled well by an exponential decay model. Roses chocolates were preferentially consumed to Quality Street chocolates in a ward setting. Chocolates were consumed primarily by healthcare assistants and nurses, followed by doctors. Further practical studies are needed.

\section{Introduction}

A higher chocolate consumption has been linked with reduced risks for the development of cardiometabolic disorders. ${ }^{1}$ The authors' experience is that chocolate consumption in a hospital environment is a relatively common occurrence, and that gifts from patients and their families represent a large proportion of healthcare workers' chocolate consumption. Subjectively, we noted that chocolate boxes emptied quickly and that determining which healthcare professionals ate the most chocolates was a common source of workplace conflict. Literature on chocolate consumption by healthcare workers in a hospital setting is lacking.

We carried out an exploratory study to provide quantitative data on patterns of chocolate consumption in a hospital environment. Specifically we estimated the survival time of a chocolate in a ward setting, modelled mathematically any pattern of chocolate consumption observed, estimated if survival time was affected by chocolate brand, and investigated differences in healthcare professionals' chocolate consumption stratified by job type. 


\section{Methods}

The study was prospective and conducted across three UK sites and four different wards: the medical assessment unit and a general medical ward at Bedford Hospital, Bedford; a haematology/oncology ward at University Hospital of North Staffordshire, Stoke-on-Trent; and a general surgical ward at Great Western Hospital, Swindon. Observations took place in the last week of August 2013. The supplementary file provides details of the protocol.

\section{On the ward}

We chose to study a $350 \mathrm{~g}$ box of Quality Street chocolates (Nestlé, Switzerland) and a $350 \mathrm{~g}$ box of Roses chocolates (Cadbury, United Kingdom), on the basis that they are commonly given as gifts and are two of the leading brands of chocolates available in the United Kingdom and elsewhere. Pilot data investigating a range of brands showed that these two contained similar numbers of chocolates (between 30 and 35 on average).

At approximately 10 am an observer (a doctor who was familiar with the ward in which testing was being carried out) covertly placed the boxes side by side in a prominent location in the main nursing or reception area, where such gifts are normally placed. The observers covertly recorded what time each box was opened, and at what time individual chocolates were taken from each box. The observers used a preprinted data collection form to record, in an anonymised fashion, the role of the person eating the chocolate (for example, doctor or nurse), ensuring that the chocolates were kept under continuous visual surveillance as much as was practical. The observation period was a minimum of two hours up to approximately four hours. Before leaving, the observers were asked to record the numbers of leftover chocolates by brand. The observers did not discuss the chocolates with ward staff, nor were they on active patient care duties during the observation periods.

\section{Outcomes}

The primary outcome was the median survival time of a chocolate. The main secondary outcome was the mean time taken for a box of chocolates to be opened when placed on the ward.

\section{Statistical analysis}

No previous studies were available on which we could base power calculations. We estimated that a total of 210 chocolates would be needed to provide $80 \%$ power $(\mathrm{P}<0.05$, two sided) to detect a $50 \%$ change in the hazard ratio between groups (Quality Street and Roses), assuming a median survival of 60 minutes for an individual chocolate, and follow-up of four hours maximum. ${ }^{2}$ Assuming a $350 \mathrm{~g}$ box of chocolates contained 30 chocolates (based on pilot data), we estimated that we would need eight boxes, totally around 240 chocolates.

Once the observers left the ward, we deemed any leftover chocolates to be "lost to follow-up." We analysed the primary outcome using Kaplan-Meier survival analysis and Cox regression. The data were also pooled and analysed separately by type of chocolate. Other continuous variables were analysed by two sided independent sample $t$ tests, with a significance level of $<0.05$. After excluding leftover chocolates, we performed regression curve fitting and estimation to generate a potential mathematical model of chocolate consumption by using statistical software, with an independent variable of survival time and a dependent variable of the proportion of chocolates remaining. We used regression curve fitting to test a variety of models (linear, logarithmic, quadratic, cubic, and exponential). The model with the highest $\mathrm{R}^{2}$ value and an $\mathrm{F}$ statistic with a two sided significance level of $<0.05$ was chosen as the best fit. Survival half life was calculated as the time taken until $50 \%$ of the chocolates remained $\left(\mathrm{t}_{1 / 2}=[\ln 0.5] / \lambda\right.$; where $\lambda$ was the decay constant). Differences in preference between the two chocolate brands (modelled as a continuous variable of percentage of total chocolate consumption) were calculated using a one sample $t$ test with a null hypothesis test value of 0.5 (that is, equal preference), repeated separately for each professional group. All statistical calculations were performed using SPSS version 20.0 (IBM Software, USA).

\section{Regulatory issues}

Ethical issues-We did not seek ethical approval for this study as no identifiable individual data were collected. We considered the effects of the availability of additional chocolate resulting in weight gain. Given the free availability of such products, lack of coercion, and that an individual's additional chocolate consumption as a result of our study was minimal, we thought this a minor issue.

Consent-No identifiable data were collected, and we did not seek prior consent from participants, as this would bias the study significantly. We weighed up the risks and benefits and thought that these were justified given that the benefits or harms to an individual of eating a chocolate were freely assessed by the individual concerned, and because no coercion took place.

\section{Results}

Overall, 191 chocolates out of a possible 258 (74\%) were observed to have been eaten. The remainder were left over and lost to follow-up. The mean observation period was 254 minutes (95\% confidence interval 179 to 329 ). No adverse events occurred. The table $\Downarrow$ summarises the ward level data.

The median survival time of a chocolate was 51 minutes $(95 \%$ confidence interval 39 to 63). Regression curve fitting suggested that the rate of emptying of a box of chocolates was best explained by an exponential decay curve with equation $\mathrm{C}_{\mathrm{p}}=\mathrm{e}-\lambda \mathrm{t}$; where $\mathrm{C}_{\mathrm{p}}$ is the proportion of chocolates remaining, $\mathrm{t}$ is the time in minutes, and $\lambda$ is the decay constant, which was -0.007 for our model (model $\left.\mathrm{R}^{2}=0.844, \mathrm{P}<0.001\right)$. Across the whole dataset, the survival half life (time taken for $50 \%$ of chocolates to be eaten) was 99 minutes.

The mean time taken for a box of chocolates to be opened after being placed on the ward was 12 minutes $(95 \%$ confidence interval 0 to 24). The time to opening of Quality Street and Roses boxes did not differ significantly (19v5 minutes, 95\% confidence interval for difference -19 to 46 minutes, $\mathrm{P}=0.35$ ). Quality Street chocolates survived longer than Roses chocolates (hazard ratio for survival of Roses $v$ Quality Street $0.70,95 \%$ confidence interval 0.53 to $0.93, \mathrm{P}=0.014$, fig $1 \Downarrow$ ).

Healthcare assistants and nurses each accounted for $28 \%$ of the total chocolates consumed ( 54 out of 191 chocolates consumed by each group). Doctors were the third biggest consumers, accounting for $15 \%$ (29 out of 191) of the total chocolates consumed. There was a trend that healthcare assistants and nurses preferred Roses chocolates, whereas doctors preferred Quality Street chocolates, but, examining each job type separately, preference for one type of chocolate was not statistically significant (fig $2 \Downarrow$ ). 


\section{Discussion}

Chocolates left on wards for staff are consumed rapidly. An exponential decay model best explained how a box of chocolates was consumed in a ward environment. Overall, ward chocolate consumers preferred Roses chocolates compared with Quality Street chocolates. Taken as a group, healthcare assistants and nurses were the largest consumers of chocolate.

To our knowledge, our multicentre, multispecialty, observational study is the first to provide quantitative data on patterns of chocolate consumption within a hospital environment. The study was appropriately powered to detect a difference between two leading chocolate brands and was conducted robustly over multiple sites, and in multiple specialty situations, to have as much external validity as possible. Bias was minimised by using a similar start time and ensuring that all observers were familiar with the study protocol. Qualitatively, the observers all commented that when a box was first opened the initial phase of chocolate consumption was rapid, followed by a steadier and ever slowing consumption as time progressed. This was confirmed by our exponential decay model, which we believe provides a sound model for these observations. The behavioural or anthropological basis of this model remains unclear and is in need of further investigation, although similar patterns are seen in a variety of biological processes.

Although we noted that healthcare assistants and nursing staff consumed a larger proportion of the total chocolates, this does not imply increased consumption per head compared with other groups. Wards have higher numbers of nurses and healthcare assistants compared with other groups, which might account for these findings. The chocolates were placed in the main ward area at the nurses' station or reception as these areas were easily accessible to all staff and the commonest place where gift chocolates are normally kept. None the less, taken as a group, the nurses and healthcare assistants consumed the majority of the chocolates.

Being novel, the study was exploratory in nature and thus had a limited sample size (although adequately powered) and observational methodology. As we explored two types of chocolate products that were artificially placed in a hospital environment we cannot comment on whether the quantitative data apply to other brands of chocolates. The observers were all doctors, which may introduce a degree of bias, although this was minimised by using a clear proforma for data capture with little observer subjectivity. ${ }^{3}$ Coincidentally, the trial was conducted during Ramadan, and so some healthcare professionals did not eat chocolate. However there are likely to be seasonal influences on chocolate consumption all year round (increased availability leading up to Christmas, decreased consumption owing to New Year dieting, increased availability leading up to Easter), so it is unclear whether there is truly any representative time for doing such research.

Differences in consumption habits were noted between ward and specialty, but we did not think that single observations were sufficient to draw conclusions on these, and instead we chose to focus on the pooled results. A larger study powered to detect differences in these endpoints would be interesting.

A meta-analysis investigated the link between chocolate consumption and cardiometabolic disorders. ${ }^{1}$ It found significant heterogeneity in the reporting of chocolate consumption, so only compared highest with lowest consuming groups. The highest consuming groups consumed chocolate more than once weekly or more than $7.5 \mathrm{~g} / \mathrm{day}$, and showed a pooled reduction in the odds of developing any cardiovascular disease and stroke. The studies included in the meta-analysis were highly variable in defining chocolate consumption and cocoa content, so it is difficult to relate these to our study findings. ${ }^{1}$ None the less, a single chocolate from our study weighed approximately $9 \mathrm{~g}$, which is above the $7.5 \mathrm{~g} /$ day threshold reported in the meta-analysis. Commercially available chocolate has a high sugar and fat content and it is likely that excessive consumption will lead to deleterious effects on population health, outweighing any potential benefits.

\section{Conclusions}

The median survival time of a chocolate in this study was just 51 minutes. $\Downarrow \Downarrow$ In keeping with many biological processes, the way a box of chocolates is consumed seems to follow an exponential decay process, with an initial rapid "grab" phase. Further appropriately powered studies investigating the preference for specific chocolate flavours over a wider variety of specialty wards may prove interesting. Given the short half life of a box of chocolates, to ensure that all healthcare staff get benefits from consistent chocolate consumption it is the authors' opinion that the frequency of chocolates delivered to wards needs to be increased and a concerted lobbying response instigated against recent manufacturers' trends in shrinking the size of chocolate boxes.

We thank the participating staff on the wards. The observers would like to apologise to anyone who received a less than truthful answer to the question: "What are you doing here?"

Contributors: PRG conceived the study, wrote the initial protocol, analysed the data, and wrote the first draft of the manuscript. He is the guarantor for the study. DJM developed the protocol, conducted experiments, collected data, and critically revised the final manuscript. PLRN collected pilot data, developed the protocol, conducted experiments, collected data, and critically revised the final manuscript. FDA and HEC conducted experiments, collected data, aided in data interpretation, and critically revised the final manuscript. RDM and CAM developed the protocol, and critically revised the final manuscript. All authors had access to the full dataset, take responsibility for the integrity of the data and the accuracy of the dataset, and gave final approval for the submission of this version for consideration of publication.

Funding: This study received no external funding. The costs of the study were borne equally by the authors. No sponsorship was obtained.

Competing interests: All authors have completed the ICMJE uniform disclosure form at www.icmje.org/coi_disclosure.pdf (available on request from the corresponding author) and declare: no support from any organisation for the submitted work; no financial relationships with any organisations that might have an interest in the submitted work in the previous three years. Other non-financial relevant interests: PRG is particularly sentimental about, and incredibly fond of, Lindt Lindor white chocolate truffles; DJM advocates abstinence as the only effective way to avoid chocolate over-consumption; PLRN is influenced by the intoxicating smells emanating from the Cadbury's chocolate factory at Bournville near his home; FDA supports her native Ghana's cocoa exports by eating a single Heroes chocolate (Cadbury) every night; HEC declares an interest in polishing off leftover Bounty chocolates (Mars); RDM's Germanic background means that he is hard-wired, like his brethren, to love all milk chocolate; and CAM reports a preference for Milkybar buttons (Nestlé).

Ethical approval: Not sought.

Data sharing: A full dataset is available from the corresponding author on request.

Transparency: PRG (guarantor) affirms that this manuscript is an honest, accurate, and transparent account of the study being reported; that no important aspects of the study have been omitted; and that any discrepancies from the study as planned have been explained. 


\section{What is already known on the topic}

Few quantitative data are available on chocolate survival in a hospital environment and on healthcare professionals' chocolate consumption, although anecdotal evidence is abundant

\section{What this study adds}

This study suggests that the survival time of a chocolate is short, at under an hour, and that the initial rate of chocolate consumption from a box is rapid but slows with time

An exponential decay model best fitted this trend

Taken as whole groups, the highest percentages of chocolates were consumed by healthcare assistants and nurses, followed by doctors

Buitrago-Lopez A, Sanderson J, Johnson L, Warnakula S, Wood A, Di Angelantonio E, et al. Chocolate consumption and cardiometabolic disorders: systematic review and meta-analysis. BMJ 2011;343:d4488.

2 Schoenfeld DA, Richter JR. Nomograms for calculating the number of patients needed for a clinical trial with survival as an endpoint. Biometrics 1982;38:163-70.

3 Podsakoff PM, MacKenzie SB, Lee JY, Podsakoff NP. Common method biases in behavioral research: a critical review of the literature and recommended remedies. J Appl Psychol 2003;88:879-903. doi:10.1037/0021-9010.88.5.879

\section{Cite this as: BMJ 2013;347:f7198}

This is an Open Access article distributed in accordance with the Creative Commons Attribution Non Commercial (CC BY-NC 3.0) license, which permits others to distribute, remix, adapt, build upon this work non-commercially, and license their derivative works on different terms, provided the original work is properly cited and the use is non-commercial. See: http://creativecommons.org/licenses/by-nc/3.0/.

Accepted: 28 November 2013 


\section{Table}

Table 1| Key data by ward

Ward specialty

Key data

Acute medical

General medical

General surgical

Haematology

Observation time (min)

360

178

246

233

Median $(95 \% \mathrm{Cl})$ survival time of chocolate $(\mathrm{min})$

96 (83 to 109)

44 (36 to 52$)$

91 (1 to 182$)$

40 (31 to 49$)$

Hazard ratio $(95 \% \mathrm{Cl})$ for survival of Roses $v$ Quality $\quad 0.92$ (0.56 to 1.51$), 0.750 .27$ (0.14 to 0.52$),<0.0010 .58$ (0.31 to 1.10$), 0.091 .70$ (0.97 to 2.99$), 0.06$

Street, $\mathrm{P}$ value 


\section{Figures}



Fig 2 Bar chart showing number and type of chocolate consumed by role of staff



Can roses really grow on you? 


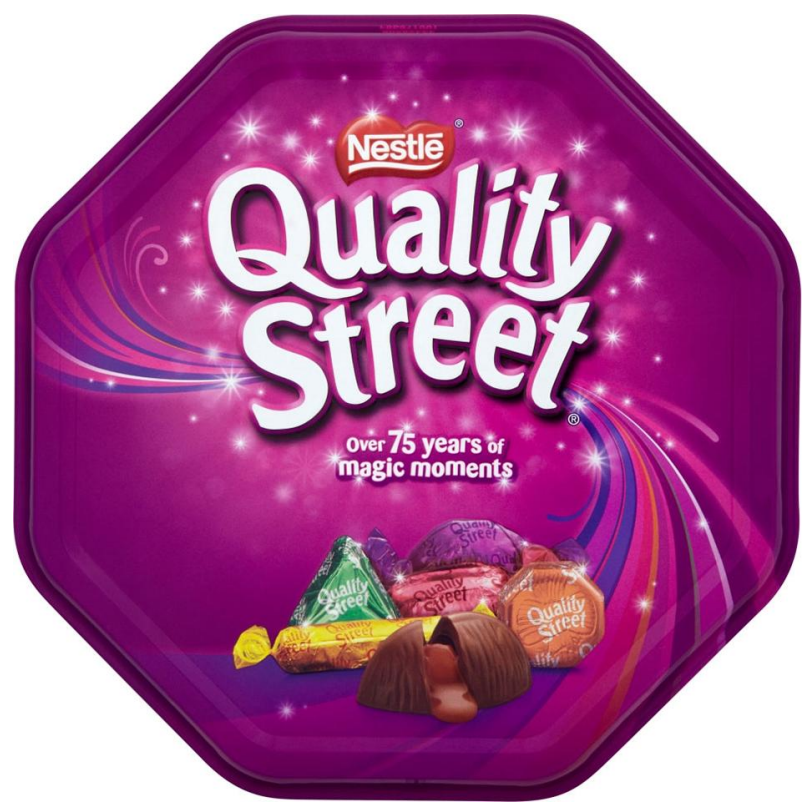

Or are these more up your street? 